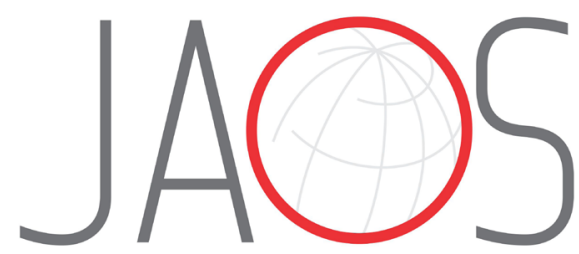

\title{
Role of Candida albicans on enamel demineralization and on acidogenic potential of Streptococcus mutans in vitro biofilms
}

\section{Abstract}

Gustavo EIDT1

Caroline Gomes de ANDRADE'

Thais de Cássia NEGRINI ${ }^{1}$

Rodrigo Alex ARTHUR ${ }^{1}$
Submitted: October 16, 2018 Modification: November 30, 2018

Accepted: December 17, 2018
There is growing evidence that $C$. albicans is associated with dental caries, but its role on caries development needs to be better clarified. Objective: To evaluate at the hard tissue level the effect of $C$. albicans on the cariogenic potential of $S$. mutans biofilms focusing on the mineral profile of induced carious lesions. This study also aimed to evaluate the effect of $C$. albicans on the acidogenic potential of $S$. mutans biofilms. Methodology: Dual-species $(\mathrm{CA}+\mathrm{SM})$ and single-species biofilms (CA or SM) were grown on the surface of enamel slabs in the presence of glucose/sucrose supplemented culture medium for 24, 48 and 72 hours. Demineralization was evaluated through percentage of surface microhardness change (\%SMC) and transversal microradiography analysis (ILM and LD) and $\mathrm{pH}$ of the spent medium was recorded daily. Data were analyzed by two-way ANOVA followed by Bonferroni correction. Results: \%SMC was statistically different among the biofilms at each time point being the highest for SM biofilms and the lowest for CA

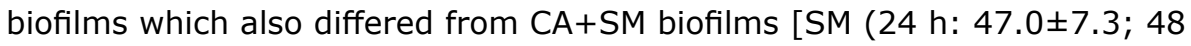

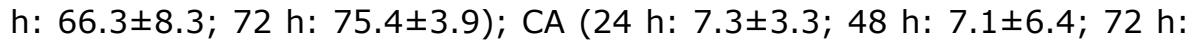

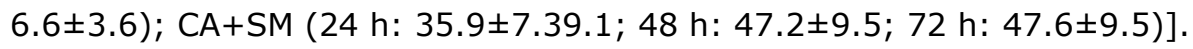
$\mathrm{pH}$ of spent medium was statistically lower for SM biofilms compared to the other biofilms at each time point and remained constant over time while $\mathrm{pH}$ values increased from 24 to $72 \mathrm{~h}$ for both CA and CA+SM biofilms [SM ( $24 \mathrm{~h}$ : $4.4 \pm 0.1 ; 48$ h: $4.4 \pm 0.1 ; 72$ h: $4.5 \pm 0.1)$; CA (24 h: $6.9 \pm 0.3 ; 48$ h: $7.2 \pm 0.2$;

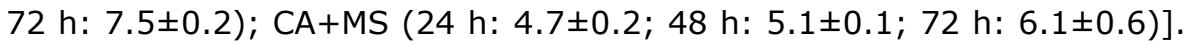
IML and LD for SM biofilms increased over time while no difference was observed from 24 to $72 \mathrm{~h}$ for the other biofilms. Conclusions: The present data suggest that $C$. albicans has low enamel demineralization potential and the presence of $C$. albicans can reduce both the cariogenic and acidogenic potentials of $S$. mutans biofilms.

Keywords: Candida albicans. Streptococcus mutans. Biofilms. Dental caries. Dental enamel.
Corresponding address: Rodrigo Alex Arthur R. Ramiro Barcelos, 2492 - Porto Alegre RS - 90035-003 - Brasil. Phone: +55 5133085193 e-mail: rodrigoarthur.ufrgs@gmail.com
${ }^{1}$ Universidade Federal do Rio Grande do Sul, Faculdade de Odontologia, Departamento de Odontologia Preventiva e Social, Porto Alegre, Rio Grande do Sul, Brasil 


\section{Introduction}

Acid production, through fermentation of carbohydrates by a selective group of microorganisms, results in $\mathrm{pH}$ reduction within biofilms. This acidification is directly related to the dissolution of tooth tissues that might occur if the balance between demineralization and remineralization process is disturbed over an extended period. ${ }^{1}$ So, in dental caries, frequent sugar intake results in a dysbiotic change in dental plaque where the acid-tolerant microorganisms prevail over the less acid-tolerant ones in response to biofilm

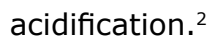

Candida albicans has a high acid tolerance and is capable of producing acids even under low $\mathrm{pH}$ conditions. These characteristics may favor the fungus in the microbial shifts associated with dental caries. ${ }^{3,4}$ In fact, the frequency of isolation of $C$. albicans is significantly higher in biofilms associated with carious teeth..$^{5-9}$

Furthermore, one of the microorganisms most frequently associated with dental caries is the bacterial species S. mutans. Despite being not alone in this task, S. mutans has a high acid production ability. In addition, it has the unique ability of converting sucrose into extracellular insoluble glucans (EPS) that constitute the main component of the biofilm matrix. Insoluble glucans alter the diffusion properties of biofilm matrix by acting as a barrier to the buffering effects of saliva. Additionally, they can promote the establishment of acidic microenvironments and thereby the formation of acidic niches that exhibit an increased demineralization potential. ${ }^{10-12}$

Literature has extensively provided evidences that biofilms formed by C. albicans and S. mutans present increased cariogenic potential compared to single-species biofilms. Previous studies have focused either on biological mechanisms related to such increased cariogenic potential and on changes induced by this microbial association on the threedimensional structure of biofilms. ${ }^{13-18}$ Rodent models have also been used to show that carious lesions produced by the association between $C$. albicans and S. mutans are more severe. ${ }^{13}$ Therefore, a positive association between the presence of $C$. albicans and the development of cariogenic biofilms may exist, especially in severe conditions such as early childhood caries. ${ }^{19-21}$

However, the role played by this microbial association on dental caries onset is still controversial since other studies have indicated opposite findings. ${ }^{22,23}$ Yet, the association between surrogate outcomes, such as the carriage of streptococci and $C$. albicans on dental plaque and saliva, and caries onset ${ }^{21}$ is questioned. ${ }^{24}$ Additionally, to the best of our knowledge, no other study has objectively assessed the profile of enamel mineral loss in the presence of that microbial association.

Thus, the aim of this study was to evaluate at the hard tissue level the effect of Candida albicans on the cariogenic potential of Streptococcus mutans biofilms focusing on the mineral profile of induced carious lesions. Additionally, this study aimed to evaluate the effect of $C$. albicans on the acidogenic potential of Streptococcus mutans biofilms. To this end, an in vitro biofilm model was used to induce enamel carious lesions that were assessed via surface hardness and whose mineral profile was evaluated by transversal microradiography. The null hypothesis was that $C$. albicans do not affect the cariogenic and acidogenic potentials of $S$. mutans biofilms.

\section{Methodology}

\section{Experimental design}

Dual-species (MIX; C. albicans + S. mutans; $n=24$ ) and single-species (C. albicans; $n=24$ or $S$. mutans; $n=24$ ) biofilms were grown on the surface of sound bovine enamel slabs in the presence of culture medium supplemented with $6 \mathrm{mM}$ glucose and $3 \mathrm{mM}$ sucrose for 24,48 and 72 hours ( $n=8$ for each biofilm experimental period). The medium was refreshed daily and the $\mathrm{pH}$ of the spent medium was recorded. Biofilms were harvested and viable cell counts were performed at the end of each experimental period. Enamel demineralization and mineral profile were evaluated through the determination of percentage of surface hardness change, via hardness analysis, and integrated mineral loss and lesion depth, via transversal microradiography. The experiments were done in triplicate.

\section{Enamel slabs preparation}

Enamel slabs were cut from sound bovine anterior teeth, which were previously disinfected in $10 \%$ formaldehyde solution, ${ }^{25}$ using a drill bench (Schulz S.A., Joinville, SC, Brazil) coupled with a $1 / 4$ inch 
diamond grit hole saw (DeWALT, Baltimore, MD, USA). The resulting $6.4 \mathrm{~mm}$ diameter enamel slabs were then flattened and polished with a grinding and polishing machine (Arotec S/A, Cotia, SP, Brazil). A 1 $\mathrm{mm}$ nail varnish strip was made on the side of each slab to create an unexposed (control) area. Slabs with cracks, scratches, exposed dentin or with mean enamel baseline hardness out of the range from 320 to 360 Vickers microhardness (VMH) were discarded. Baseline surface hardness determination is described below. Slabs were then randomized and balanced into experimental groups by mean VMH values. Finally, the slabs were mounted on individual nylon cylinders attached to the lid of 24 -well plates ${ }^{26}$ and they were subjected to hydrogen peroxide gas sterilization. ${ }^{27}$

\section{Inoculum preparation}

Candida albicans (ATCC 90028) and Streptococcus mutans (UA 159) were cultivated from frozen stocks on Brain Heart Infusion Agar (BHI; Kasvi, São José dos Pinhais, PR, Brazil). Colony-forming units (CFU) were then inoculated into tubes containing Tryptic Soy Broth (TSB; HiMedia, Mumbai, MH, India baseline $\mathrm{pH}$ from 7.1 to $7.3 ; 14 \mathrm{mM}$ glucose basal concentration) supplemented with $12.5 \mathrm{mM}$ sucrose. After $24 \mathrm{~h}$ of incubation, aliquots of each suspension were transferred into fresh medium and were further incubated for $24 \mathrm{~h}$ at $37^{\circ} \mathrm{C}$. Optical density was adjusted independently for each microorganism to $0.5 \pm 0.1$ at $550 \mathrm{~nm}$ with a spectrophotometer (Milton Roy Co., Ivyland, PA, USA), corresponding to approximately $10^{7} \mathrm{CFU} / \mathrm{mL}$ of $C$. albicans and $10^{8} \mathrm{CFU} / \mathrm{mL}$ of $S$. mutans. Counts of viable cells were confirmed at each experiment by plating the inoculum on $\mathrm{BHI}$ agar plates and incubating them under the conditions described above. Inoculum presented a mixture of hyphae and yeasts forms (qualitatively determined by microscopic analysis). Dual-species consortium was obtained by combining equal amounts of the density-adjusted cultures ${ }^{26}$.

\section{In vitro biofilm growth}

The modified lids with the slabs were put onto 24well plates with $0.4 \mathrm{~mL}$ inoculum and $1 \mathrm{~mL}$ TSB $(\mathrm{pH} 7$; supplemented with $6 \mathrm{mM}$ glucose and $3 \mathrm{mM}$ sucrose; TSBGS; $20 \mathrm{mM}$ glucose final concentration) on each well and kept at $37^{\circ} \mathrm{C}$. After $8 \mathrm{~h}$ of incubation, the slabs were transferred to new plates containing $1.4 \mathrm{~mL}$ of TSBGS medium and incubated at $37^{\circ} \mathrm{C}$ overnight. Biofilms were then transferred every 24 hours to new 24-well plates containing fresh TSBGS medium and kept at $37^{\circ} \mathrm{C}^{26}$. The $\mathrm{pH}$ values of the spent medium (at 8, 24, 48 and $72 \mathrm{~h}$ ) were individually measured for each well using a $\mathrm{pH}$ meter (Digimed, São Paulo, $\mathrm{SP}$, Brazil) previously calibrated with $\mathrm{pH} 7.0$ and $\mathrm{pH}$ 4.0 standards.

\section{Biofilm harvesting and viable cell counts}

At the end of each experimental period (after 24, 48 and $72 \mathrm{~h}$ of biofilm growth), enamel slabs $(n=8)$ were aseptically removed from the lids and individually transferred to tubes containing $1 \mathrm{~mL}$ of sterile $0.9 \%$ $\mathrm{NaCl}$ and 4 sterile glass beads (4-5 mm diameter). Tubes were vortexed for $30 \mathrm{~s}$ to disperse the biofilms. Aliquots of the microbial suspensions were serially diluted and plated on BHI agar. Plates were incubated at $37^{\circ} \mathrm{C}$ under microaerophilic conditions for $24 \mathrm{~h}$. CFU were then counted under a stereomicroscope and the results expressed as CFU/mL. Enamel slabs were gently cleaned and stored in pre-codified vials at $4^{\circ} \mathrm{C}$ under humid environment until further assessment of caries lesion development.

\section{Surface microhardness}

Baseline enamel surface hardness was determined by a microhardness tester (ISH-TDV2000; Insize Co. Ltd, Suzhou, JS, China) by making five Vickers indentations, spaced $100 \mu \mathrm{m}$ from each other, with a load of $200 \mathrm{gf}$ for 10 seconds. After each experimental period of biofilm growth, five indentations were placed $100 \mu \mathrm{m}$ at the right side of the baseline indentations following the same parameters described above. The mean Vickers microhardness (VMH) values of baseline (B) and post-biofilm (P) VMH were averaged and the percentage of surface microhardness change (\% SMC) was calculated as ${ }^{26} \% \mathrm{SMC}=[(P-B) \div B] \times 100$.

\section{Transversal microradiography (TMR)}

After post-biofilm hardness analysis, half of the specimens were sectioned through the center with a low-speed diamond saw (Isomer; Buehler Ltd., Lake Bluff, IL, USA), perpendicularly to the surface and transversally to the varnish strip, in order to obtain slices approximately $150 \mu \mathrm{m}$ thick representing both exposed and unexposed (sound) enamel surfaces. Slices were then hand polished plane-parallel from both cut sides with 600 and 1200 grit sandpapers to a thickness of approximately $100 \mu \mathrm{m}$. Specimens were mounted in a custom-made sample-holder with an aluminium calibration step wedge with 14 steps. Microradiographs were taken with an X-ray generator 
(Softex Co. Ltd., Ebina, Japan) on a high precision glass plate (Konica Minolta Inc., Tokyo, Japan) at a distance of $42 \mathrm{~cm}$ using $20 \mathrm{kV}$ and $20 \mathrm{~mA}$ for 13 minutes. Plates were developed for $5 \mathrm{~min}$, rinsed with deionized water and fixed for $8 \mathrm{~min}$ in a dark room at $20^{\circ} \mathrm{C}$. All plates were then washed in running water for $10 \mathrm{~min}$ and air-dried. Microradiographs were examined using a microscope (Carl Zeiss Microscopy GmbH, Jena, TH, Germany) in conjunction with a camera (Canon, Tokyo, Japan) and a computer running data-acquisition and calculation software programs (Inspektor Research Inc., Amsterdam, NH, Netherlands). The lesion depth (LD) was calculated using a threshold of $95 \%$ of the mineral content of sound enamel. Integrated mineral loss (IML; vol\% mineral $x \mu \mathrm{m}$ ) was also calculated. ${ }^{28}$ All analyses were performed by a blinded examiner.

\section{Statistical analysis}

The mean and standard deviation ( $\mathrm{pH}$ of spent medium, \% SMC, LD and IML) were calculated for each tested condition. The assumptions of homogeneity of variances and normality of the distribution were checked. Counts of viable cells were log transformed and were descriptively reported as median and quartiles. Two-way analysis of variance (ANOVA) was used to determine the effects and interactions of the tested conditions (biofilm microbial composition and biofilm age) on response variables. Bonferroni adjusted comparisons were used when two-way ANOVA indicated statistically significant effects. Correlation between $\mathrm{pH}$ and counts of fungal viable cells on dual-species biofilms was tested using Pearson's coefficient, while correlation between \%SMC and

Table 1- Viable cell counts [Log CFU/mL; median ( $25^{\text {th }} / 75^{\text {th }}$ quartiles)] according to biofilm age and biofilm microbial composition

\begin{tabular}{|c|c|c|c|c|c|}
\hline \multirow{3}{*}{ Variable } & \multicolumn{5}{|c|}{ Biofilm microbial composition } \\
\hline & \multirow[t]{2}{*}{ Age } & \multicolumn{2}{|c|}{ C. albicans } & \multicolumn{2}{|c|}{ MIX } \\
\hline & & $\begin{array}{c}\text { Median } \\
\left(25^{\text {th }}-75^{\text {th }}\right)\end{array}$ & $\mathbf{n}$ & $\begin{array}{c}\text { Median } \\
\left(25^{\text {th }}-75^{\text {th }}\right)\end{array}$ & $\mathbf{n}$ \\
\hline \multirow[t]{3}{*}{ Fungi } & $24 \mathrm{~h}$ & $6.4(6.3-6.7)$ & 24 & $6.3(6.0-6.4)$ & 24 \\
\hline & $48 \mathrm{~h}$ & $6.6(6.3-7.2)$ & 24 & $6.6(6.4-6.7)$ & 24 \\
\hline & $72 \mathrm{~h}$ & $7.1(6.5-7.5)$ & 24 & $6.7(6.6-7.1)$ & 24 \\
\hline \multirow[t]{5}{*}{ Bacteria } & & S. mutans & & MIX & \\
\hline & & $\begin{array}{c}\text { Median } \\
\left(25^{\text {th }}-75^{\text {th }}\right)\end{array}$ & $\mathbf{n}$ & $\begin{array}{c}\text { Median } \\
\left(25^{\text {th }}-75^{\text {th }}\right)\end{array}$ & $\mathbf{n}$ \\
\hline & $24 \mathrm{~h}$ & $8.0(7.9-8.1)$ & 24 & $7.9(7.7-8.0)$ & 24 \\
\hline & $48 \mathrm{~h}$ & $8.0(7.4-8.1)$ & 24 & $7.7(7.3-8.0)$ & 24 \\
\hline & $72 \mathrm{~h}$ & $8.1(7.7-8.2)$ & 24 & $7.8(7.2-8.2)$ & 24 \\
\hline
\end{tabular}

Table 2- Spent medium pH, percentage of Surface Microhardness Change (\%SMC), Integrated Mineral Loss (IML; Vol\% x $\mu \mathrm{m})$ and Lesion Depth (LD; $\mu \mathrm{m})$ according to biofilm age and biofilm microbial composition

\begin{tabular}{|c|c|c|c|c|c|c|c|}
\hline \multirow{3}{*}{ Variable } & \multirow{3}{*}{ Age } & \multicolumn{6}{|c|}{ Biofilm microbial composition } \\
\hline & & \multicolumn{2}{|c|}{ C. albicans } & \multicolumn{2}{|c|}{ MIX } & \multicolumn{2}{|c|}{ S. mutans } \\
\hline & & Mean \pm SD & $\mathbf{n}$ & Mean \pm SD & $\mathbf{n}$ & Mean \pm SD & $\mathbf{n}$ \\
\hline \multirow[t]{3}{*}{$\mathrm{pH}$} & $24 \mathrm{~h}$ & $6.9 \pm 0.3^{\mathrm{Aa}}$ & 24 & $4.7 \pm 0.2^{\mathrm{Ab}}$ & 24 & $4.4 \pm 0.1^{\mathrm{Ac}}$ & 24 \\
\hline & $48 \mathrm{~h}$ & $7.2 \pm 0.2^{\mathrm{Ba}}$ & 24 & $5.1 \pm 0.2^{\mathrm{Bb}}$ & 24 & $4.4 \pm 0.1^{\mathrm{Ac}}$ & 24 \\
\hline & $72 \mathrm{~h}$ & $7.5 \pm 0.2^{\mathrm{Ca}}$ & 24 & $6.1 \pm 0.6^{\mathrm{cb}}$ & 24 & $4.5 \pm 0.1^{\mathrm{Ac}}$ & 24 \\
\hline \multirow[t]{3}{*}{$\% S M C$} & $24 \mathrm{~h}$ & $7.3 \pm 3.3^{\mathrm{Aa}}$ & 24 & $35.9 \pm 9.1^{\mathrm{Ab}}$ & 24 & $47.0 \pm 7.3^{\mathrm{AC}}$ & 24 \\
\hline & $48 \mathrm{~h}$ & $7.1 \pm 6.4^{\mathrm{Aa}}$ & 24 & $47.2 \pm 9.5^{\mathrm{Bb}}$ & 24 & $66.3 \pm 8.3^{\mathrm{Bc}}$ & 24 \\
\hline & $72 \mathrm{~h}$ & $6.6 \pm 3.6^{\mathrm{Aa}}$ & 24 & $47.6 \pm 9.5^{\mathrm{Bb}}$ & 24 & $75.4 \pm 3.9^{\mathrm{cc}}$ & 24 \\
\hline \multirow[t]{3}{*}{ IML } & $24 \mathrm{~h}$ & $211.4 \pm 60.9^{\mathrm{Aa}}$ & 7 & $310.0 \pm 140.8^{\mathrm{Aa}}$ & 7 & $391.7 \pm 146.5^{\text {Аa }}$ & 6 \\
\hline & $48 \mathrm{~h}$ & $276.7 \pm 155.1^{\mathrm{Aa}}$ & 9 & $667.5 \pm 182.7^{\text {Aab }}$ & 8 & $1172.9 \pm 478.3^{\mathrm{Bb}}$ & 7 \\
\hline & $72 \mathrm{~h}$ & $238.6 \pm 130.6^{\mathrm{Aa}}$ & 7 & $596.3 \pm 199.0^{\mathrm{Aa}}$ & 8 & $2025.6 \pm 862.9^{\mathrm{cb}}$ & 9 \\
\hline \multirow[t]{3}{*}{ LD } & $24 \mathrm{~h}$ & $11.4 \pm 6.0^{\mathrm{Aa}}$ & 7 & $15.1 \pm 5.7^{\mathrm{Aa}}$ & 7 & $19.6 \pm 7.2^{\mathrm{Aa}}$ & 6 \\
\hline & $48 \mathrm{~h}$ & $18.9 \pm 16.8^{\mathrm{Aa}}$ & 9 & $31.9 \pm 9.5^{\text {Aab }}$ & 8 & $67.9 \pm 60.2^{\mathrm{ABD}}$ & 7 \\
\hline & $72 \mathrm{~h}$ & $13.3 \pm 7.7^{\mathrm{Aa}}$ & 7 & $32.0 \pm 11.8^{\mathrm{Aa}}$ & 8 & $103.0 \pm 56.7^{\mathrm{Bb}}$ & 9 \\
\hline
\end{tabular}

Different uppercase letters show a significant difference between biofilm age and different lowercase letters show a significant difference between biofilm microbial compositions by two-way ANOVA followed by Bonferroni test $(p<0.01)$ 
transversal microradiography outcomes was tested with Spearman's rank coefficient. All analyses were performed on SPSS Statistics for Windows, version 22.0 (IBM Corp. in Armonk, NY, USA) with a significance level set as $1 \%$.

\section{Results}

Regarding counts of viable cells, only changes lower than $1 \log _{10}$ (10-fold) were found on median of CFU among the different tested conditions for both fungal and bacterial cells. Overall, CFU remained constant among different periods of biofilm growth irrespective to the biofilm composition (Table 1). For all the other outcomes ( $\mathrm{pH}, \% \mathrm{SMC}$, IML and LD) statistical analysis indicated an interaction effect between microbial composition and biofilm age $(p<0.01)$

The mean of $\mathrm{pH}$ values after 8 hours of biofilm growth was $6.7 \pm 0.2,4.8 \pm 0.1$ and $4.4 \pm 0.1$ for C. albicans, dual-species and $S$. mutans biofilms respectively. Spent medium $\mathrm{pH}$ related to $S$. mutans biofilms remained between 4.4 - 4.5 under all the tested experimental conditions being significantly lower than for other biofilms at each time point. The $\mathrm{pH}$ of dual-species biofilms increased over time being below the critical $\mathrm{pH}$ for enamel demineralization for up to $48 \mathrm{~h}$ of biofilm growth. Under all the tested conditions, $\mathrm{pH}$ of the spent medium of $C$. albicans biofilms slightly increased over time but remained higher than the critical $\mathrm{pH}$ needed to induce enamel carious lesions (Table 2). A moderate correlation between fungi CFU and spent medium $\mathrm{pH}(\mathrm{r}=0.559 ; \mathrm{p}<0.01)$ was found in dual-species biofilms.
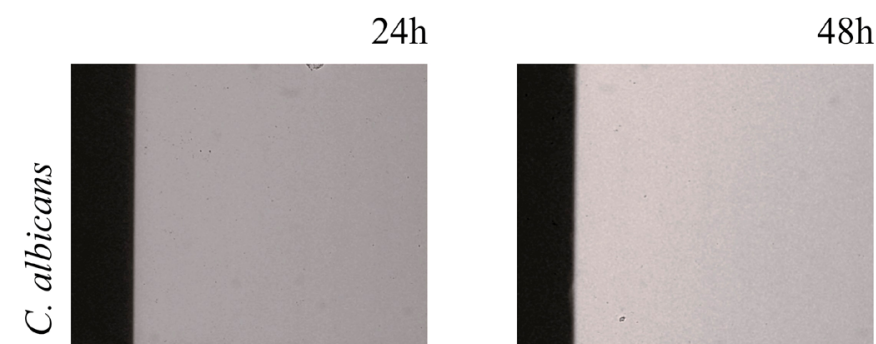

$48 \mathrm{~h}$
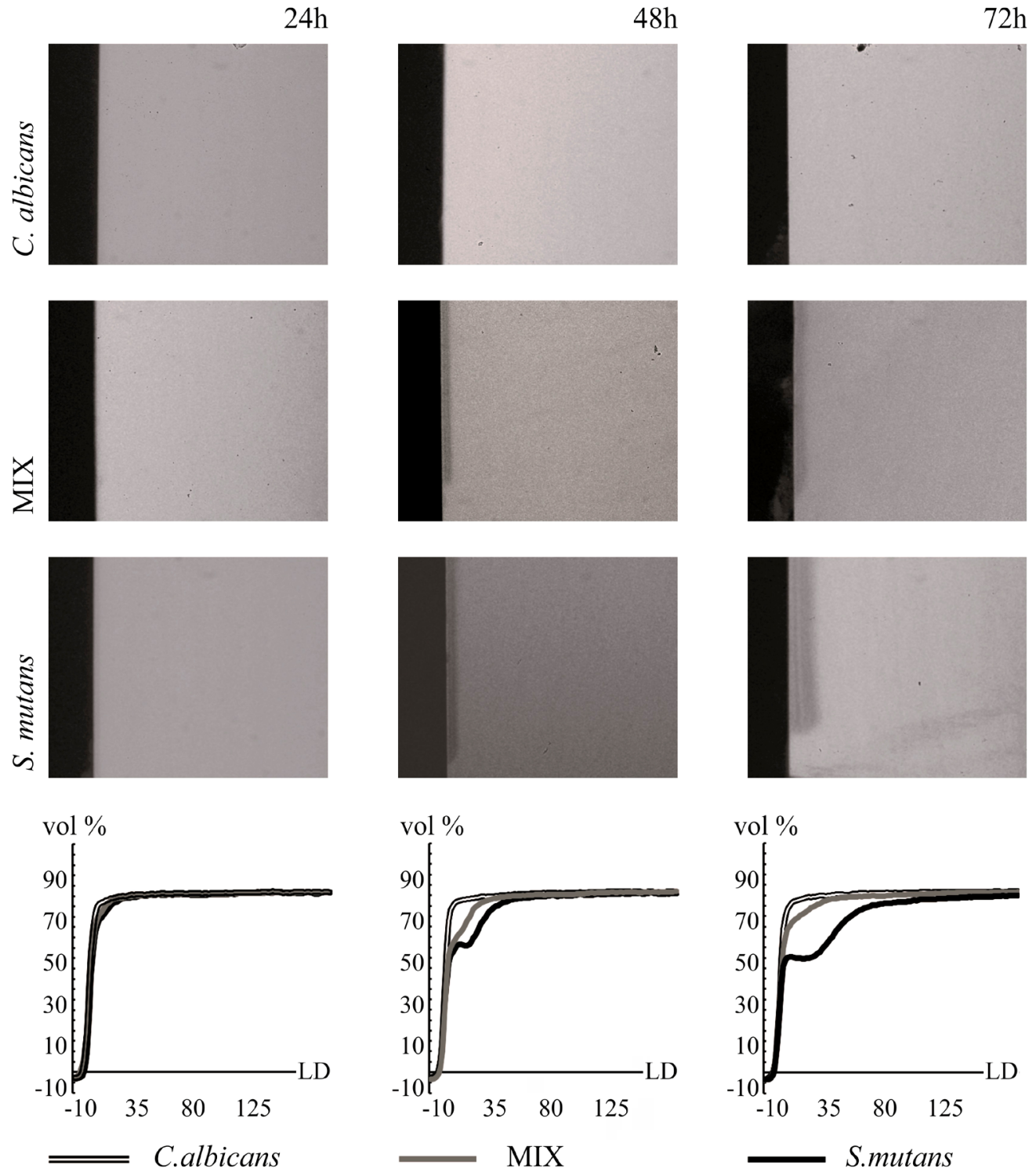

Figure 1- Representative microradiographs and mineral profile after 24, 48 and 72 hours of biofilm growth. Different coloured lines represent mean values of the percentage of mineral volume (vol \%) according to lesion depth (LD) for each biofilm microbial composition 
In relation to carious lesion development, higher $\%$ SMC was found in the presence of $S$. mutans singlespecies biofilms than for other biofilms at each time point. In addition, $S$. mutans related demineralization increased with time, being statistically different among the different time points. On the other hand, carious lesion development in the presence of $C$. albicans single-species biofilms was statistically lower than in the presence of other biofilms and it remained constant over time. Additionally, the \% SMC in the presence of dual-species biofilms at $48 \mathrm{~h}$ of biofilm formation was statistically higher than at $24 \mathrm{~h}$, but it was not statistically different compared to enamel demineralization after $72 \mathrm{~h}$ of biofilm growth (Table 2 ).

At $24 \mathrm{~h}$ of biofilm growth, no statistical difference was found on IML and LD among the different biofilms, but enamel slabs exposed to $S$. mutans single-species biofilms presented IML and LD higher than slabs exposed to $C$. albicans single-species biofilms at 48 and $72 \mathrm{~h}$ and higher than dual-species biofilms at 72 $\mathrm{h}$ of biofilm growth. Furthermore, IML of enamel slabs exposed to $S$. mutans biofilms increased over time, being statistically different at each time point, whereas LD at $72 \mathrm{~h}$ was statistically higher than that found at $24 \mathrm{~h}$ of biofilm growth. Moreover, IML of enamel slabs exposed to C. albicans and to dual-species biofilms remained constant over time. This same behaviour was observed in relation to LD (Table 2).

Figure 1 shows representative transversal microradiographs of enamel slabs according to the experimental conditions where it can be easily seen that all carious lesions were sub-superficial presenting a well-mineralized surface layer. Lesions formed in the presence of $S$. mutans biofilms were deeper and had lower mineral content compared to the other biofilms. Overall, a positive correlation was found between $\%$ SMC and IML $(\rho=0.808 ; p<0.01)$ as well as between $\%$ SMC and LD $(\rho=0.760 ; p<0.01)$.

\section{Discussion}

As the presence of $C$. albicans has been increasingly related to dental caries, ${ }^{21}$ the present study used an in vitro biofilm model for the assessment of enamel carious lesion development at the hard tissue level by using surface hardness and transversal microradiography aiming to investigate the effect of $C$. albicans on the cariogenic potential of $S$. mutans biofilms as well as on their acidogenic potential.

It is well known that $S$. mutans, in addition to its acid-tolerance and acidogenic potential, can produce exoenzymes, known as glucosyltransferase B (GtfB), that synthesize EPS from sucrose, an essential factor associated to the cariogenic potential of biofilms. ${ }^{10,12,29}$ So, as expected, enamel slabs exposed to $S$. mutans single-species biofilms presented an increased mineral loss over time, taking both superficial microhardness and transversal microradiography outcomes into consideration (Table 2, Figure 1). On the other hand, less acidification was found in the presence of $C$. albicans single-species biofilms which lead to very little enamel demineralization (Table 2, Figure 1).

This low acidogenic potential found in C. albicans single-species biofilms could be related to the fact that this fungus may present a more complex acid production profile than lactic acid-producing bacteria. ${ }^{3}$ Pyruvate and acetate may be produced by this fungus in the presence of glucose alone, but almost no spent medium acidification has been found for $C$. albicans single-species biofilms grown in the presence of glucose and sucrose. ${ }^{23}$ Chemical characterization of $C$. albicans single-species related spent medium suggests minimum carbohydrate utilization and a poor ability of the fungus in metabolizing sucrose when growing alone. ${ }^{17}$ Additionally, it has been also suggested that in the presence of culture medium supplemented with sucrose, C. albicans may produce ethanol that does not influence the $\mathrm{pH}$ of the medium. ${ }^{23}$ Important to mention that the less acidified environment found in the presence of $C$. albicans could not be attributed to any colonization impairment or lack of fungi viability, since, although slightly changes had been found over time, CFU counts remained constant and similar to those of $S$. mutans throughout the experiments (Table 1 ).

It is important to acknowledge though that the low cariogenicity of $C$. albicans single-species biofilms found in this study might be strain-related and might also be a result of different biofilm growing conditions and/or experimental conditions compared to other studies since there are evidences showing that biofilms of $C$. albicans strains isolated from HIV+ children can cause enamel demineralization after 5 days of biofilm growth ${ }^{30,31}$ and that carious lesions are developed in rats infected with C. albicans. ${ }^{13,22}$ Nonetheless, it seems based on the present data that the isolated cariogenic potential of $C$. albicans in relation to enamel is not high.

Carbon source has a direct influence on biofilm 
morphogenesis. ${ }^{32-34}$ When C. albicans and S. mutans are growing together in mixed biofilms, they may compete for fermentable sugars. Under carbohydrate privation, C. albicans can use a variety of other carbon sources, such as amino acids, fatty acids and carboxylic acids. ${ }^{35}$ Breakdown of exogenous amino acids can lead to extrusion of ammonia, a highly basic compound. Consequently, in a glucose-limited milieu, C. albicans can actively modulate extracellular $\mathrm{pH}$ by alkalinisation of acidic environments. ${ }^{36}$

Additionally, C. albicans consumption of organic acids as carbon sources, such as pyruvate and lactate, is also responsible for the neutralization of acidic environments. It is likely that both mechanisms are acting together leading to the reduced acidification of dual-species biofilms over time. This way, in the presence of dual-species biofilms enamel slabs showed an initial caries lesion development, but no additional mineral loss could be observed after 48 hours (Figure 1). By providing data related to surface hardness and mineral profile of demineralized enamel specimens, our results corroborate with the results of Willems, et al. ${ }^{23}$ (2016) reinforcing that C. albicans reduce the cariogenic potential of $S$. mutans biofilms. It is important to highlight that Willems's study and ours used similar biofilm growth conditions (up to 72 hours in a medium with high protein and low carbohydrate contents). Thus, this reduced cariogenic potential may only occur in environments with high nutritional content but low carbohydrate concentrations.

One may argue that the reduced cariogenicity found on dual-species biofilms was a result of the less carbohydrate availability for biofilm growth, since the same sugar concentration was used for both singleand dual-species biofilms. It is important to emphasize though that the use of higher sugar concentration would lead to enamel surface softening compromising the assessment of enamel demineralization by means of surface hardness. Even having plenty of available sugars, C. albicans as single-species biofilm was not able to induce considerable enamel demineralization suggesting that the available sugar concentration was not the reason related to the reduced cariogenicity of dual-species biofilms. Moreover, it could also be argued that the increase on $\mathrm{pH}$ of spent medium of dual-species biofilms would be the result of a reduction on viable cells counts over time. It is important to emphasize that changes on counts of viable cells were too small (less than $1 \log _{10}$-fold) and do not present any relevance for caries development.

However, the data of the present study differ from previous studies. A more aggressive onset of carious lesions was found in the presence of dual-species biofilms. ${ }^{13}$ It has been shown that GtfB secreted by S. mutans adheres to $C$. albicans yeast cell surface being kept under an enzymatically active form promoting the formation of EPS-rich biofilm matrix and enhancing accumulation of $S$. mutans on biofilms. ${ }^{37}$ Yet, C. albicans-bound GtfB produces more EPS than $S$. mutans-bound exoenzyme, leading to an enhanced EPS accumulation on biofilm matrix. ${ }^{14}$ On the other hand, downregulation of bacterial exoenzymes can occur in the presence of $C$. albicans leading to lack of EPS production by $S$. mutans ${ }^{38}$ which could also reduce the cariogenic potential of $S$. mutans biofilms. ${ }^{22}$ It is unclear though at this time the effect on three-dimensional structure of biofilms imposed by the present dualspecies biofilm model. Further studies are necessary to assess the impact of the tested model and of the tested growth conditions on biofilm matrix production and its relation to carious lesion development.

\section{Conclusion}

Overall, the data of the present study suggest that the presence of $C$. albicans can reduce the cariogenic potential of $S$. mutans biofilms when there is a competition for nutrients. Under this condition, $C$. albicans also modulates spent medium pH decreasing the acidogenic potential of $S$. mutans biofilms.

\section{Conflicts of interest statement}

The authors declare to have no conflicts of interest in the subject matter discussed in this manuscript.

\section{Acknowledgements}

The authors would like to thank CAPES (Coordination for the Improvement of Higher Education Personnel) which conceived a scholarship to the first and second authors. This study is derived from a thesis submitted by the first author to the Dental School of Federal University of Rio Grande do Sul (UFRGS) as partial fulfillment of the requirements for a Master's Degree in Clinical Dentistry - Cariology/Restorative Dentistry. Partial results were presented at the $64^{\text {th }}$ ORCA Congress, held in Oslo (Norway), 2017 


\section{References}

1- Takahashi N, Nyvad B. The role of bacteria in the caries process: ecological perspectives. J Dent Res. 2011;90(3):294-303.

2- Mira A, Simon-Soro A, Curtis MA. Role of microbial communities in the pathogenesis of periodontal diseases and caries. J Clin Periodontol. 2017;44(S18):S23-38.

3- Klinke T, Kneist S, Soet JJ, Kuhlisch E, Mauersberger S, Forster A, et al. Acid production by oral strains of Candida albicans and lactobacilli. Caries Res. 2009;43(2):83-91.

4- Pereira D, Seneviratne CJ, Koga-Ito CY, Samaranayake LP. Is the oral fungal pathogen Candida albicans a cariogen? Oral Dis. 2017;24(4):518-26.

5- Fragkou S, Balasouli C, Tsuzukibashi O, Argyropoulou A, Menexes G, Kotsanos N, et al. Streptococcus mutans, Streptococcus sobrinus and Candida albicans in oral samples from caries-free and caries-active children. Eur Arch Paediatr Dent. 2016;17(5):367-75.

6- Naidu BV, Reginald BA. Quantification and correlation of oral Candida with caries index among different age groups of school children: a casecontrol study. Ann Med Health Sci Res. 2016;6(2):80-4.

7- Carvalho FG, Silva DS, Hebling J, Spolidorio LC, Spolidorio DM. Presence of mutans streptococci and Candida spp. in dental plaque/ dentine of carious teeth and early childhood caries. Arch Oral Biol. 2006;51(11):1024-8.

8- Yang XQ, Zhang Q, Lu LY, Yang R, Liu Y, Zou J. Genotypic distribution of Candida albicans in dental biofilm of Chinese children associated with severe early childhood caries. Arch Oral Biol. 2012;57(8):1048-53.

9- Moraga CP, Rodríguez Martínez GA, Lefimil Puente CA, Morales Bozo IC, Urzúa Orellana BR. Prevalence of Candida albicans and carriage of Candida non-albicans in the saliva of preschool children, according to their caries status. Acta Odontol Scand. 2016;75(1):30-5.

10- Bowen WH, Koo H. Biology of Streptococcus mutans-derived glucosyltransferases: role in extracellular matrix formation of cariogenic biofilms. Caries Res. 2011;45(1):69-86.

11- Bowen WH, Burne RA, Wu H, Koo H. Oral biofilms: pathogens, matrix, and polymicrobial interactions in microenvironments. Trends Microbiol. 2017;26(3):229-42.

12- Koo H, Falsetta ML, Klein MI. The exopolysaccharide matrix: a virulence determinant of cariogenic biofilm. J Dent Res. 2013;92(12):1065-73. 13- Falsetta ML, Klein MI, Colonne PM, Scott-Anne K, Gregoire S, Pai $\mathrm{C}-\mathrm{H}$, et al. Symbiotic relationship between Streptococcus mutans and Candida albicans synergizes virulence of plaque biofilms in vivo. Infect Immun. 2014;82(5):1968-81.

14- Hwang G, Marsh G, Gao L, Waugh R, Koo H. Binding force dynamics of Streptococcus mutans-glucosyltransferase B to Candida albicans. J Dent Res. 2015;94(9):1310-7.

15- Hwang G, Liu Y, Kim D, Li Y, Krysan DJ, Koo H. Candida albicans mannans mediate Streptococcus mutans exoenzyme GtfB binding to modulate cross-kingdom biofilm development in vivo. PLoS Pathog. 2017;13(6):e1006407.

16- Ellepola K, Liu Y, Cao T, Koo H, Seneviratne CJ. Bacterial GtfB augments Candida albicans accumulation in cross-kingdom biofilms. J Dent Res. 2017;96(10):1129-35.

17- Kim D, Sengupta A, Niepa THR, Lee B-H, Weljie A, Freitas-Blanco VS, et al. Candida albicans stimulates Streptococcus mutans microcolony development via cross-kingdom biofilm-derived metabolites. Sci Rep. $2017 ; 7: 41332$.

18- He J, Kim D, Zhou X, Ahn S-J, Burne RA, Richards VP, et al. RNAseq reveals enhanced sugar metabolism in Streptococcus mutans co-cultured with Candida albicans within mixed-species biofilms. Front Microbiol. 2017;8:1036.

19- Qiu R, Li W, Lin Y, Yu D, Zhao W. Genotypic diversity and cariogenicity of Candida albicans from children with early childhood caries and cariesfree children. BMC Oral Health. 2015;15(1):144.
20- Li W, Yu D, Gao S, Lin J, Chen Z, Zhao W. Role of Candida albicanssecreted aspartyl proteinases (Saps) in severe early childhood caries. Int J Mol Sci. 2014;15(6):10766-79.

21- Xiao J, Huang X, Alkhers N, Alzamil H, Alzoubi S, Wu TT, et al. Candida albicans and early childhood caries: a systematic review and meta-analysis. Caries Res. 2018;52(1-2):102-12.

22- Klinke T, Guggenheim B, Klimm W, Thurnheer T. Dental caries in rats associated with Candida albicans. Caries Res. 2011;45(2):100-6. 23- Willems M, Kos K, Jabra-Rizk MA, Krom BP. Candida albicans in oral biofilms could prevent caries. Pathog Dis. 2016;74(5):1-6.

24- Twetman S. Current controversies - is there merit? Adv Dent Res. 2009;21(1):48-52.

25- Western JS, Dicksit DD. A systematic review of randomized controlled trials on sterilization methods of extracted human teeth. J Conserv Dent. 2016;19(4):343-6.

26- Arthur RA, Waeiss RA, Hara AT, Lippert F, Eckert GJ, Zero DT. A defined-multispecies microbial model for studying enamel caries development. Caries Res. 2013;47(4):318-24.

27- Arthur RA, Martins VB, Oliveira CL, Leitune VC, Collares FM, Magalhães $A C$, et al. Effect of over-the-counter fluoridated products regimens on root caries inhibition. Arch Oral Biol. 2015;60(10):1588-94. 28- Salomão PM, Comar LP, Buzalaf MA, Magalhães AC. In situ remineralisation response of different artificial caries-like enamel lesions to home-care and professional fluoride treatments. BMC Oral Health. 2016;16(1):2.

29- Xiao J, Klein MI, Falsetta ML, Lu B, Delahunty CM, Yates JR $3^{\text {rd }}$, et al. The exopolysaccharide matrix modulates the interaction between 3D architecture and virulence of a mixed-species oral biofilm. PLoS Pathog. 2012;8(4):e1002623.

30- Charone S, Portela MB, Chagas MS, Araújo Soares RM, Araújo Castro GF. Biofilm of Candida albicans from oral cavity of an HIV-infected child: challenge on enamel microhardness. Oral Surg Oral Med Oral Pathol. 2013;115(4):500-4

31- Charone S, Portela MB, Martins KO, Soares RM, Castro GF. Role of Candida species from HIV infected children in enamel caries lesions: an in vitro study. J Appl Oral Sci. 2017;25(1):53-60.

32- Ten Cate JM, Klis FM, Pereira-Cenci T, Crielaard W, de Groot PW. Molecular and cellular mechanisms that lead to Candida biofilm formation. J Dent Res. 2009;88(2):105-15.

33- Brambilla E, Ionescu AC, Cazzaniga G, Ottobelli M, Samaranayake LP. Levorotatory carbohydrates and xylitol subdue Streptococcus mutans and Candida albicans adhesion and biofilm formation. J Basic Microbiol. 2016;56(5):480-92

34- Jin Y, Samaranayake LP, Samaranayake Y, Yip HK. Biofilm formation of Candida albicans is variably affected by saliva and dietary sugars. Arch Oral Biol. 2004;49(10):789-98.

35- Danhof HA, Vylkova S, Vesely EM, Ford AE, Gonzalez-Garay M, Lorenz MC. Robust extracellular $\mathrm{pH}$ modulation by Candida albicans during growth in carboxylic acids. MBio. 2016;7(6):e01646-16.

36- Vylkova S, Carman AJ, Danhof HA, Collette JR, Zhou H, Lorenz MC. The fungal pathogen Candida albicans autoinduces hyphal morphogenesis by raising extracellular pH. MBio. 2011;2(3):e00055-11. 37- Gregoire S, Xiao J, Silva BB, Gonzalez I, Agidi PS, Klein MI, et al. Role of glucosyltransferase $B$ in interactions of Candida albicans with Streptococcus mutans and with an experimental pellicle on hydroxyapatite surfaces. Appl Environ Microbiol. 2011;77(18):6357-67. 38- Sztajer H, Szafranski SP, Tomasch J, Reck M, Nimtz M, Rohde M, et al. Cross-feeding and interkingdom communication in dual-species biofilms of Streptococcus mutans and Candida albicans. ISME J. 2014;8(11):2256-71. 